\author{
F. F. Bellotti • M. T. Yamashita
}

\title{
Single Particle Momentum Distributions for Three-Bosons in Two and Three Dimensions and Dimensional Crossover
}

Received: 23 March 2015 / Accepted: 7 August 2015 / Published online: 21 August 2015

(C) Springer-Verlag Wien 2015

\begin{abstract}
In this paper, we review our main results involving the single particle momentum distribution of bosonic trimer states in two and three dimensions. A summary table makes easier the comparison between the matrix elements and the different terms of the momentum distributions. We also show a practical method to continuously interpolate between different dimensions.
\end{abstract}

\section{Introduction}

Many nature laws can be strongly affected if dimensionality is changed. As already pointed out by Landau in his classic book [1], any infinitesimal amount of attraction produce a bound state in $2 \mathrm{D}$, while a finite amount of attraction is necessary to bind a 3D system. A remarkable phenomenon related to the dimensionality of the system arises in the study of three identical bosons, where the differences in the energy spectrum (and also other observables) are directly related to the number of dimensions that this system may access: in 2D there are only two three-body bound states linked to one two-body bound state in the limit where the range of the potential goes to zero [2]. On the other hand, in 3D, the number of three-body bound states may grow to infinity $[3,4]$ - this effect is now called by Efimov effect.

The Efimov effect corresponds to an accumulation of the three-boson energy levels, toward zero energy, when the two-body scattering length tends to infinity. In this limit, where the two-body energy is zero, the energies of successive states are geometrically spaced obeying a universal ratio. These states were predicted and observed for three identical bosons in 3D systems [5,6], but are absent in 2D even in the most favorable scenario of mass-imbalanced systems [7,8], where a mass-dependent effective potential favors the binding of a light particle to a heavy dimer [9].

The appearance of Efimov states in 3D is very closely related to the possibility of collapse the three-body system. This collapse (Thomas collapse), firstly derived by Thomas in 1935, says that the three-body ground

F. F. Bellotti $(\varangle)$

Department of Physics and Astronomy, Aarhus University, 8000 Aarhus C, Denmark

E-mail: bellotti@phys.au.dk

Tel.: +458715 5595

Fax: +4586120740

F. F. Bellotti

Instituto Tecnológico de Aeronáutica, São José dos Campos, SP 12228-900, Brazil.

F. F. Bellotti

Instituto de Fomento e Coordenação Industrial, São José dos Campos, SP 12228-901, Brazil.

M. T. Yamashita

Instituto de Física Teórica, UNESP-Univ Estadual Paulista, C.P. 70532-2, São Paulo, CEP 01156-970, Brazil 
state energy may be made as deep as you want by decreasing the range of the potential $\left(r_{0}\right)$-in the limit $r_{0} \rightarrow 0$ the three-body binding energy tends to infinity. This divergence demands the inclusion of a cutoff or, equivalently, a new physical scale independent of the two-body energy. In 2D, this collapse is absent in such a way the three-body observables are proportional to the two-body energy. For example, the three-body ground state energy is $16.52 E_{2}$ and the energy of the first excited state is $1.267 E_{2}$ for three identical bosons [2].

There are many examples of observables in cold atomic gases that are affected by the dimensionality of the system. We would like to start mentioning the two- $\left(C_{2}\right)$ and three-body $\left(C_{3}\right)$ contact parameters. The connection between universal two-body correlations to many-body properties through the quantity $C_{2}$ was proposed by Tan [10] (this quantity is often called Tan's contact parameter). For example, the variation in the energy of a Fermi gas of momentum $k_{F}$ with the interaction strength (scattering length $a$ ) is directly proportional to this $C_{2}$, namely

$$
2 \pi \frac{\mathrm{d} E}{\mathrm{~d}\left[-1 /\left(k_{F} a\right)\right]}=C_{2} .
$$

Furthermore, the virial theorem for this atomic gas also relates with $C_{2}$ through

$$
E-2 V=-\frac{C_{2}}{4 \pi k_{F} a}
$$

These relations, in the way they are presented, were confirmed in experiments with two-component Fermi gases [11], where each side of Eqs. (1) and (2) were measured independently and after compared to each other. A later experiment showed that similar relations also hold for bosons [12].

The quantities on the left-hand-side of Eqs. (1) and (2) are defined through the many-body properties of the gas, while the contact parameter is defined in the few-body sector. A way to determine this parameter is to find the coefficient in the leading order of the asymptotic one-body large momentum density, $n(q)$, of few-body systems, given by

$$
\lim _{q \rightarrow \infty} n(q) \rightarrow \frac{C_{2}}{q^{4}}+C_{3} F(q)+\cdots .
$$

The next order in this expansion defines the three-body contact parameter, $C_{3}$, which may be important only for bosonic systems, since the Pauli principle suppresses the short-range correlations for two-component Fermi gases. Notice that the momentum dependence of the leading order term in this expansion is the same for 1D, 2D and 3D systems [13], but the function $F(q)$ depends on the dimensionality of the system [14]. The twoand three-body contact parameters were determined for three identical bosons in 2D [14] and 3D [15], and for mixed-species systems in 2D [16] and 3D [17].

An interesting point about the two-body contact parameter is that, despite the considerable difference between the binding energy of both states for three identical bosons in 2D (where the well-known limit cycle is not present), the ratio $\frac{C_{2}}{E_{3}}$ is the same for the two states [14]. In general, for a mixed-species system-which have a richer energy spectrum $[9,18]-$ the ratio $\frac{C_{2}}{E_{3}}$ is not the same for all states, but only in the special and experimentally accessible case of a three-body system composed for at least two identical non-interacting particles [16].

Among the several differences involving the dimensionality of the system we would like to stress that the function $F(q)$ in Eq. (3) has very distinct forms in each case of 2D or 3D. This function is directly related to the spectator functions $f(q)$ [given in Eqs. (4) and (5)], whose asymptotic form were discovered in the 60's for 3D systems [19] and approximately 50 years later for 2D systems $[14,16]$. Thus, an interesting question is whether it is possible to interpolate between the $3 \mathrm{D}$ and $2 \mathrm{D}$ limits in a simple theoretical way and subsequently explore this in simulations using both more involved numerical methods and experimental setups since the development of the techniques for cooling and trap atoms allows the interpolation between different dimensions [20,21].

We proposed a model that has the ability to interpolate geometrically between two and three spatial dimensions and thus study this crossover for both two- and three-body bound states of identical bosons. A "squeezed" dimension, whose size can be varied to interpolate the two limits, is employed with periodic boundary conditions (PBC). This model has the unique feature that it can be regularized analytically, which is a great advantage for its numerical implementation allowing to go smoothly between both limits. The theoretical elegance and tractability of calculations in the three-body system is itself a strong incentive for pursuing this geometry, but in spite of this elegance, a direct connection between experiments and the parameter that dials between different dimensions with PBC in this model was not found yet. 
Table 1 Matrix elements of the two-body T-matrix, $\tau_{\alpha}^{D}\left(q, E_{3}\right)$ and the kernels $K_{\alpha \beta}^{D}\left(q, k, E_{3}\right)$ and $K_{\alpha \gamma}^{D}\left(q, k, E_{3}\right)$ for both 2D and 3D systems, where $\mu$ is the subtraction point (see, for example, [22])

2D $3 \mathrm{D}$

\begin{tabular}{llr}
\hline$\left.\tau_{\alpha}^{D}\left(q, E_{3}\right)\right]^{-1}$ & $4 \pi m_{\beta \gamma} \ln \left(\sqrt{\frac{\frac{q^{2}}{2 m_{\beta \gamma, \alpha}}-E_{3}}{\left|E_{\beta \gamma}\right|}}\right)$ & $\pi\left(2 m_{\beta \gamma}\right)^{3 / 2}\left(\sqrt{\left(\frac{q^{2}}{2 m_{\beta \gamma, \alpha}}-E_{3}\right)}-\sqrt{\left|E_{\beta \gamma}\right|}\right)$ \\
$K_{\alpha \beta}^{D}\left(q, k, E_{3}\right)$ & $\frac{1}{\sqrt{\left(-E_{3}+\frac{q^{2}}{2 m_{\alpha \gamma}}+\frac{k^{2}}{2 m_{\beta \gamma}}\right)^{2}-\left(\frac{k q}{m_{\gamma}}\right)^{2}}}$ & $\frac{m_{\gamma}}{q}\left(\ln \frac{-E_{3}+\frac{q^{2}}{2 m_{\alpha \gamma}}+\frac{k^{2}}{2 m_{\beta \gamma}}+\frac{k q}{m_{\gamma}}}{-E_{3}+\frac{q^{2}}{2 m_{\alpha \gamma}}+\frac{k^{2}}{2 m_{\beta \gamma}}-\frac{k q}{m_{\gamma}}}-\ln \frac{\mu^{2}+\frac{q^{2}}{2 m_{\alpha \gamma}}+\frac{k^{2}}{2 m_{\beta \gamma}}+\frac{k q}{m_{\gamma}}+\frac{q^{2}}{2 m_{\alpha \gamma}}+\frac{k^{2}}{2 m_{\beta \gamma}}-\frac{k q}{m_{\gamma}}}{m_{\alpha \gamma}}\right)$ \\
$K_{\alpha \gamma}^{D}\left(q, k, E_{3}\right)$ & $\frac{1}{\sqrt{\left(-E_{3}+\frac{q^{2}}{2 m_{\alpha \beta}}+\frac{k^{2}}{2 m_{\beta \gamma}}\right)^{2}-\left(\frac{k q}{m_{\beta}}\right)^{2}}}$ & $\frac{m_{\beta}}{q}\left(\ln \frac{-E_{3}+\frac{q^{2}}{2 m_{\alpha \beta}}+\frac{k^{2}}{2 m_{\beta \gamma}}+\frac{k q}{m_{\beta}}}{-E_{3}+\frac{q^{2}}{2 m_{\alpha \beta}}+\frac{k^{2}}{2 m_{\beta \gamma}}-\frac{k q}{m_{\beta}}}-\ln \frac{\mu^{2}+\frac{q^{2}}{2 m_{\alpha \beta}}+\frac{k^{2}}{2 m_{\beta \gamma}}+\frac{k q}{m_{\beta}}}{\mu^{2}+\frac{q^{2}}{2 m_{\alpha \beta}}+\frac{k^{2}}{2 m_{\beta \gamma}}-\frac{k q}{m_{\beta}}}\right)$ \\
\hline
\end{tabular}

In the next sections we review our main results involving the momentum distributions in three and two dimensions. Both results are put together side-by-side in a table where the comparison becomes easier. In the last section we give an overview of our method that continuously interpolates between 3 and 2D limits.

\section{Integral Equation for Bound States}

We investigate $a b c$ bound systems whose dynamics is restricted to either two (2D) or three spatial dimensions (3D). The masses are $m_{a}, m_{b}, m_{c}$ and the pairwise interactions are described for attractive zero-range potentials, being $E_{a b}, E_{a c}, E_{b c}$ the energy of each pair. The three-body wave function $\left\langle\mathbf{q}_{\alpha}, \mathbf{p}_{\alpha} \mid \Psi_{a b c}\right\rangle$ has the same functional form in both $2 \mathrm{D}$ and $3 \mathrm{D}$. For any $s$-wave bound state, the energy $E_{3}$ is a solution of the free Schrödinger equation, except in the region where particles overlap. Using the Faddeev decomposition in momentum space, the bound state wave function in units of $\hbar=1$ is written as

$$
\left\langle\mathbf{q}_{\alpha}, \mathbf{p}_{\alpha} \mid \Psi_{a b c}\right\rangle=\Psi\left(\mathbf{q}_{\alpha}, \mathbf{p}_{\alpha}\right)=\frac{f_{\alpha}\left(q_{\alpha}\right)+f_{\beta}\left(\left|\mathbf{p}_{\alpha}-\frac{m_{\beta}}{m_{\beta}+m_{\gamma}} \mathbf{q}_{\alpha}\right|\right)+f_{\gamma}\left(\left|\mathbf{p}_{\alpha}+\frac{m_{\gamma}}{m_{\beta}+m_{\gamma}} \mathbf{q}_{\alpha}\right|\right)}{\left|E_{3}\right|+\frac{q_{\alpha}^{2}}{2 m_{\beta \gamma, \alpha}}+\frac{p_{\alpha}^{2}}{2 m_{\beta \gamma}}},
$$

where $\alpha, \beta, \gamma$ are cyclic permutations of $a, b, c, \mathbf{q}_{\alpha}$ is the $\alpha$ particle momenta with respect to the $\mathrm{CM}$ of the pair $\beta \gamma, \mathbf{p}_{\alpha}$ is the pair relative momenta, $m_{\beta \gamma, \alpha}=m_{\alpha}\left(m_{\beta}+m_{\gamma}\right) /\left(m_{\alpha}+m_{\beta}+m_{\gamma}\right)$ and $m_{\beta \gamma}=\left(m_{\beta}+m_{\gamma}\right) /\left(m_{\beta}+\right.$ $\left.m_{\gamma}\right)$ are the reduced masses and $f_{\alpha, \beta, \gamma}(\mathbf{q})$ are the Faddeev components, or spectator functions. The three-body energy, $E_{3}$, and the spectator functions $f_{\alpha, \beta, \gamma}$ are solution of a set of three coupled homogeneous integral equations, which in a compact form reads

$$
f_{\alpha}(\mathbf{q})=\tau_{\alpha}^{D}\left(q, E_{3}\right) \int_{0}^{\infty} k d k\left[K_{\alpha \beta}^{D}\left(q, k, E_{3}\right) f_{\beta}(k)+K_{\alpha \gamma}^{D}\left(q, k, E_{3}\right) f_{\gamma}(k)\right],
$$

where the matrix elements of the two-body T-matrix, $\tau_{\alpha}^{D}\left(q, E_{3}\right)$ and the kernels $K_{\alpha \beta}^{D}\left(q, k, E_{3}\right)$ and $K_{\alpha \gamma}^{D}\left(q, k, E_{3}\right)$ are given in Table 1.

An interesting difference between 2D and 3D three-body systems can be seen in Eq. (5) and Table 1. For each non-interacting $\beta \gamma$ pair, the respective spectator function $f_{\alpha}(q)=0$. Choosing $E_{\beta \gamma}=0$ in the first line of Table 1 gives different result for $2 \mathrm{D}$ and $3 \mathrm{D}$ systems. In $3 \mathrm{D},\left[\tau_{\alpha}^{D}\left(q, E_{3}\right)\right]^{-1}$ is finite and Eq. (5) is well-defined even if the three two-body subsystems interact with zero energy. On the other hand in 2D, $\left[\tau_{\alpha}^{D}\left(q, E_{3}\right)\right]^{-1} \rightarrow \infty$, meaning that if at least two pairs have zero energy, Eq. (5) is not well-defined and three-body bound states do not exist. Therefore, non-interacting and zero-energy two-body systems lead to the same result in 2D, while they can give completely different results in 3D [17].

\section{Momentum Distribution}

The one-body density functions are observable quantities even in the limit of large momenta where the number of atoms is small, which has already been observed in experiments using time-of-flight and the mapping to momentum space [23], Bragg spectroscopy [11] or momentum-resolved photo-emission spectroscopy [24]. 
The one-body momentum density of the particle $\alpha$ is defined through the wave function $\Psi\left(\mathbf{q}_{\alpha}, \mathbf{p}_{\alpha}\right)$ from Eq. (4) as

$$
n\left(q_{\alpha}\right)=\int d^{D} p_{\alpha}\left|\Psi\left(\mathbf{q}_{\alpha}, \mathbf{p}_{\alpha}\right)\right|^{2}
$$

and the normalization is $\int d^{D} q_{\alpha} n\left(q_{\alpha}\right)=1$, where $D=2,3$ for $2 \mathrm{D}$ or $3 \mathrm{D}$ systems, respectively.

Inserting Eq. (4) in Eq. (6) and expanding it, the nine initial terms can be grouped into four components by using arguments of symmetry, each one with a distinctly different integrand structure. The one-body momentum density is expressed as a sum of this four terms, i.e., $n\left(q_{\alpha}\right)=\sum_{i=1}^{4} n_{i}\left(q_{\alpha}\right)$.

A general system of three distinguishable particles, presents three distinct one-body momentum distributions, each one corresponding to a different particle. The four terms for particle $\alpha$ are expressed as

$$
\begin{aligned}
& n_{1}\left(q_{\alpha}\right)=\left|f_{\alpha}\left(q_{\alpha}\right)\right|^{2} \int d^{D} k \frac{1}{\left(-E_{3}+\frac{q_{\alpha}^{2}}{2 m_{\beta \gamma, \alpha}}+\frac{k^{2}}{2 m_{\beta \gamma}}\right)^{2}}, \\
& n_{2}\left(q_{\alpha}\right)=\int d^{D} k \frac{\left|f_{\beta}(k)\right|^{2}}{\left(-E_{3}+\frac{q_{\alpha}^{2}}{2 m_{\alpha \gamma}}+\frac{k^{2}}{2 m_{\beta \gamma}}+\frac{\mathbf{k} \cdot \mathbf{q}_{\alpha}}{m_{\gamma}}\right)^{2}}+\int d^{D} k \frac{\left|f_{\gamma}(k)\right|^{2}}{\left(-E_{3}+\frac{q_{\alpha}^{2}}{2 m_{\alpha \beta}}+\frac{k^{2}}{2 m_{\beta \gamma}}-\frac{\mathbf{k} \cdot \mathbf{q}_{\alpha}}{m_{\beta}}\right)^{2}}, \\
& n_{3}\left(q_{\alpha}\right)=f_{\alpha}^{*}\left(q_{\alpha}\right) \int d^{D} k\left[\frac{f_{\gamma}(k)}{\left(-E_{3}+\frac{q_{\alpha}^{2}}{2 m_{\alpha \gamma}}+\frac{k^{2}}{2 m_{\beta \gamma}}+\frac{\mathbf{k} \cdot \mathbf{q}_{\alpha}}{m_{\gamma}}\right)^{2}}+\frac{f^{2}}{\left(-E_{3}+\frac{q_{\alpha}^{2}}{2 m_{\alpha \beta}}+\frac{k^{2}}{2 m_{\beta \gamma}}-\frac{\mathbf{k} \cdot \mathbf{q}_{\alpha}}{m_{\beta}}\right)^{2}}\right]+c . c ., \\
& n_{4}\left(q_{\alpha}\right)=\int d^{D} \frac{f_{\beta}\left(\left|\mathbf{k}-\frac{m_{\beta}}{m_{\beta}+m_{\gamma}} \mathbf{q}_{\alpha}\right|\right) f_{\gamma}^{*}\left(\left|\mathbf{k}+\frac{m_{\gamma}}{m_{\beta}+m_{\gamma}} \mathbf{q}_{\alpha}\right|\right)}{\left(-E_{3}+\frac{q_{\alpha}^{2}}{2 m_{\beta \gamma, \alpha}}+\frac{p^{2}}{2 m_{\beta \gamma}}\right)^{2}}+\text { c.c. }
\end{aligned}
$$

Notice that the distributions for the other particles are obtained by cyclic permutations of $(\alpha, \beta, \gamma)$ in these expressions.

Although the equations for bound states (Eq. 5) and momentum distributions (Eqs. 7-10) were derived for a general case of three distinguishable particles, we now specialize to experimentally relevant systems composed by two identical bosons $a$ and a distinct particle $b$. The large momentum limit of Eqs. (7)-(10) were derived in detail for 2D and 3D systems respectively in $[16,17]$ and the final result for the density profile of particle $b$ with respect to the pair $a a$ is presented in Table 2 , in units of $m_{a}=1$.

In the following we discuss two interesting properties in the momentum distribution of $a a b$ systems in both 2D and 3D. The geometric scaling of the Efimov states implies that observables may be described independently of the quantum state. The independence of the quantum states is not expected to be valid for $2 \mathrm{D}$ systems, since

Table 2 Asymptotic forms for both 2D and 3D systems where $\mathcal{A}=\frac{m_{b}}{m_{a}}, \tan \theta_{3}=\sqrt{\frac{\mathcal{A}+2}{\mathcal{A}}}$ for $0 \leq \theta_{3} \leq \pi / 2$ and $\tan \theta_{4}=$ $\sqrt{\mathcal{A}(\mathcal{A}+2)}$ for $0 \leq \theta_{4} \leq \pi / 2, \Gamma, c_{a}$ and $c_{b}$ are normalization constants and $n_{5}\left(q_{b}\right)$ is the second order term in the expansion of $n_{2}\left(q_{b}\right)$ in Eq. (8)

\begin{tabular}{llc}
\hline & $2 \mathrm{D}$ & $3 \mathrm{D}$ \\
\hline$n_{1}\left(q_{b}\right)$ & $16 \pi \frac{\mathcal{A}}{\mathcal{A}+2} \Gamma^{2} \frac{\ln ^{2}\left(q_{b}\right)}{q_{b}^{6}}$ & $\frac{\pi^{2}\left|c_{b}\right|^{2}}{q_{b}^{5}} \sqrt{\frac{\mathcal{A}}{\mathcal{A}+2}}$ \\
$n_{2}\left(q_{b}\right)$ & $\frac{32 \pi}{q_{b}^{4}} \frac{\mathcal{A}^{2}}{(\mathcal{A}+1)^{2}} \int_{0}^{\infty} d k k\left|f_{a}(k)\right|^{2}$ \\
$n_{3}\left(q_{b}\right)$ & $\frac{4 \pi^{2} c_{a} c_{b}}{q_{b}^{5} \cosh \left(\frac{s \pi}{2}\right)}\left\{\sqrt{\frac{\mathcal{A}}{\mathcal{A}+2}} \cos \left(s \ln \sqrt{\left.\frac{\mathcal{A}+1}{2 \mathcal{A}}\right) \cosh \left[s\left(\frac{\pi}{2}-\theta_{3}\right)\right]}\right.\right.$ & $\left.+\sin \left(s \ln \sqrt{\frac{\mathcal{A}+1}{2 \mathcal{A}}}\right) \sinh \left[s\left(\frac{\pi}{2}-\theta_{3}\right)\right]\right\}$ \\
& $32 \pi \frac{\mathcal{A}}{\mathcal{A}+1} \Gamma^{2} \frac{\ln ^{3}\left(q_{b}\right)}{q_{b}^{6}}$ & $\frac{8 \pi^{2}\left|c_{a}\right|^{2}}{s q_{b}^{5} \cosh \left(\frac{s \pi}{2}\right)} \frac{\mathcal{A}^{2}}{\sqrt{\mathcal{A}(\mathcal{A}+2)}}\left\{\sqrt{\mathcal{A}(\mathcal{A}+2)} \sinh \left[s\left(\frac{\pi}{2}-\theta_{4}\right)\right]\right.$ \\
$n_{4}\left(q_{b}\right)$ & $8 \pi \Gamma^{2} \frac{\ln ^{3}\left(q_{b}\right)}{q_{b}^{6}}$ & $\left.-\frac{s \mathcal{A}}{\mathcal{A}+1} \cosh \left[s\left(\frac{\pi}{2}-\theta_{4}\right)\right]\right\}$ \\
$n_{5}\left(q_{b}\right)$ & $\frac{32 \pi}{3} \frac{\mathcal{A}(\mathcal{A}-2)}{(\mathcal{A}+1)^{2}} \Gamma^{2} \frac{\ln ^{3}\left(q_{b}\right)}{q_{b}^{6}}$ & $-\frac{8 \pi^{2}\left|c_{a}\right|^{2}}{q_{b}^{5}} \frac{\mathcal{A}^{3}(\mathcal{A}+3)}{(\mathcal{A}+1)^{3} \sqrt{\mathcal{A}(\mathcal{A}+2)}}$ \\
\hline
\end{tabular}




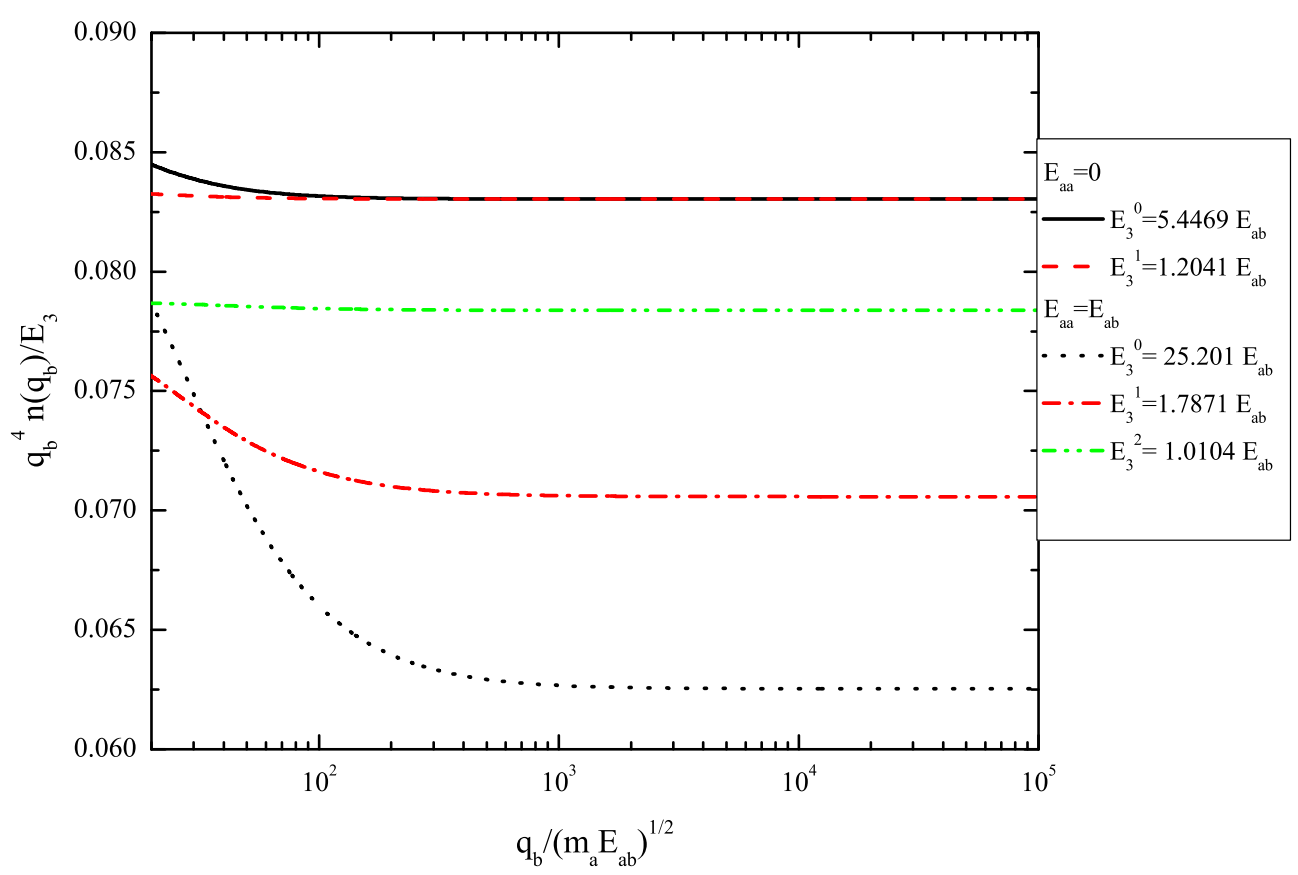

Fig. 1 The leading order term of the one-body momentum density divided by $E_{3}^{n}$ for each bound state labeled as $n$ in a system composed of two identical $\left(a={ }^{40} \mathrm{~K}\right)$ particles and a distinct one $\left(b={ }^{6} \mathrm{Li}\right)$ as a function of the momentum q for both $E_{a a}=E_{a b}$ and $E_{a a}=0$

they do not present any geometric scaling. However, the leading order in the large momentum distribution was found to be independent of the state for three identical bosons [14,25] and for $a a b$ systems, if the $a a$ subsystem is not interacting [16].

The effect of the two-body energy on the contact parameter is shown in Fig. 1 for the ${ }^{40} \mathrm{~K}^{40} \mathrm{~K}^{6} \mathrm{Li}$ system. This system has three excited states when $E_{a a}=E_{a b}$ and only two when $E_{a a}=0$. Notice that the large momentum limit of the momentum density goes to a constant in all cases. For $E_{a a}=0$ both momentum distributions are equal in units of the three-body energy, i.e., $n^{0}\left(q_{b}\right) / E_{3}^{0}=n^{1}\left(q_{b}\right) / E_{3}^{1}$, where the superscript denotes the quantum state. This case is rather special because the two identical particles have zero energy and cannot provide a scale such that the three-body structure is determined by the identical two-body interactions in the identical subsystems. In other words the large-momentum limit of the one-body density for particle $a$ is determined by the properties of the $a b$ subsystem.

This picture changes when $E_{a a}=E_{a b}$, as seen in Fig. 1. Now, in the large-momentum limit, the coefficients of the one-body densities change with the excitation energy. The systematics is that the coefficients move towards the corresponding values for $E_{a a}=0$ as function of excitation energy. First the differences of the ratios with the two-body energies is understandable, since the interaction of the two identical particles now must affect the three-body structure at small distances, and hence at large momenta. However, as the three-body binding energy decreases, the size of the system increases and details of the short-distance structure becomes less important.

The independence of the state in the one-body momentum distribution of 2D three-body system can happen or not, depending on the energy of the two-body subsystem. However, both mass-imbalanced and identical bosons systems present the same functional form to the leading order (LO) and next-to-leading order(NLO) in the large momentum expansion of the one-body density. The same does not happens in 3D, since the NLO contribution due to non-oscillatory terms vanishes when $\mathcal{A}=0.20,1.00$ and 1.57, i.e., the sum of the components $n_{1}\left(q_{b}\right), n_{3}\left(q_{b}\right), n_{4}\left(q_{b}\right), n_{5}\left(q_{b}\right)$ from Table 2 is null for these mass ratios [17].

We want to emphasize that both the results for 2D and 3D can be experimentally checked in the near future, since the calculations were made for alkali atoms. Besides the identical mass case, the other two mass ratios used in the $3 \mathrm{D}$ calculations can be obtained with mixtures of ${ }^{133} \mathrm{Cs}$ and ${ }^{87} \mathrm{Rb}(\mathcal{A}=1.565)$ or ${ }^{6} \mathrm{Li}$ and ${ }^{39} \mathrm{~K}$ $(\mathcal{A}=0.179)$. 


\section{3D: 2D Transition with PBC}

The physical and mathematical differences of three-body systems restricted to either 2D or 3D, presented in the previous sections, are the motivation that lead us to pursuit a method where the dimensionality enters as a parameter allowing to continuously interpolate between the well-known extremes of $2 \mathrm{D}$ and $3 \mathrm{D}$. We restrict analyses to three-identical bosons, which presents the Efimov effect in 3D, but only two three-body bound states in 2D. Furthermore, the dimensionality plays an important role in the momentum distribution already in this simplest case, as can be seen in Table 2 for $\mathcal{A}=1$.

Periodic boundary conditions (PBC) are assumed to be valid for the distance between the particles in the $z$ direction. The relative momentum is given by $\mathbf{p}_{\perp}=\left(p_{x}, p_{y}\right)$ in the flat $2 \mathrm{D}$ surface and by

$$
p_{z}=\frac{\sqrt{2} \pi n}{L}=\frac{n}{R}, \quad n=0, \pm 1, \pm 2, \ldots,
$$

in the transverse direction, with $L=\sqrt{2} \pi R$ being the size of the compact dimension corresponding to a radius $R$, which is the parameter that dials between two and three-dimensions. When $R \rightarrow 0$ it selects the 2D case and in the opposite limit, i.e., $R \rightarrow \infty$, the 3D case is selected [26]. The momentum $\mathbf{p}$ and its corresponding phase factor $d \mathbf{p}$ are, with PBC, defined as

$$
p^{2}=p_{\perp}^{2}+\frac{n^{2}}{R^{2}} \quad \text { and } \quad d \mathbf{p}=\frac{1}{R} d^{2} p_{\perp} .
$$

We introduce the symbol $\mathcal{f}$, which indicates an integration over the continuum momentum in the plane $\left(p_{\perp}\right)$ and a sum over the discrete perpendicular momentum $\left(p_{z}=\frac{n}{R}\right)$. It reads

$$
\mathcal{Y} d \mathbf{p} \equiv \sum_{n=-\infty}^{\infty} \int \frac{1}{R} d^{2} p_{\perp}
$$

Using definition (12), the three-body free Hamiltonian becomes

$$
\begin{aligned}
H_{0}^{p}(\mathbf{q}, \mathbf{k}) & =\left(\mathbf{q}_{\perp}+\mathbf{q}_{z}\right)^{2}+\left(\mathbf{k}_{\perp}+\mathbf{k}_{z}\right)^{2}+\left(\mathbf{q}_{\perp}+\mathbf{q}_{z}\right) \cdot\left(\mathbf{k}_{\perp}+\mathbf{k}_{z}\right), \\
& =q_{\perp}^{2}+k_{\perp}^{2}+\mathbf{q}_{\perp} \cdot \mathbf{k}_{\perp}+\frac{n^{2}}{R^{2}}+\frac{m^{2}}{R^{2}}+\frac{n m}{R^{2}}
\end{aligned}
$$

and considering Eqs. (12)-(14), the integral equation for the bound state (5) for a compact dimension with $\mathrm{PBC}$ is found to be

$$
f\left(\mathbf{q}_{\perp}, n\right)=-\frac{2}{R} \tau_{p}\left[\frac{3}{4}\left(q_{\perp}^{2}+\frac{n^{2}}{R^{2}}\right)-E_{3}\right] \sum_{m=-\infty}^{\infty} \int d^{2} k_{\perp}\left(\frac{f\left(\mathbf{k}_{\perp}, m\right)}{-E_{3}+H_{0}^{p}(\mathbf{q}, \mathbf{k})}-\frac{f\left(\mathbf{k}_{\perp}, m\right)}{\mu^{2}+H_{0}^{p}(\mathbf{q}, \mathbf{k})}\right)
$$

with $\tau_{p}(E)$ given in Eq. (16) and $H_{0}^{p}(\mathbf{q}, \mathbf{k})$ in Eq. (14). The subtraction is kept even after the discretization because the Thomas collapse is always present for any finite compact radius, no matter how small it is. It is worthwhile to remind that, for $R \rightarrow \infty$, Eq. (15) returns precisely the equation for the spectator function in 3D (5).

The two-body scattering amplitude is

$$
\tau_{p}(E)^{-1}=-\frac{2 \pi}{R} \ln \left[\frac{\sinh (\pi R \sqrt{|E|})}{\sinh \left(\pi R \sqrt{\left|E_{2}\right|}\right)}\right],
$$

which recovers the matrix elements of 3D and 2D systems in the limits $R \rightarrow \infty$ and $R \rightarrow 0$, respectively. The first case is straightforward an reads

$$
\tau_{3 D}^{-1}(E)=\lim _{R \rightarrow \infty} \tau_{p}^{-1}(E)=-2 \pi^{2}\left(\sqrt{|E|}-\sqrt{\left|E_{2}\right|}\right) .
$$




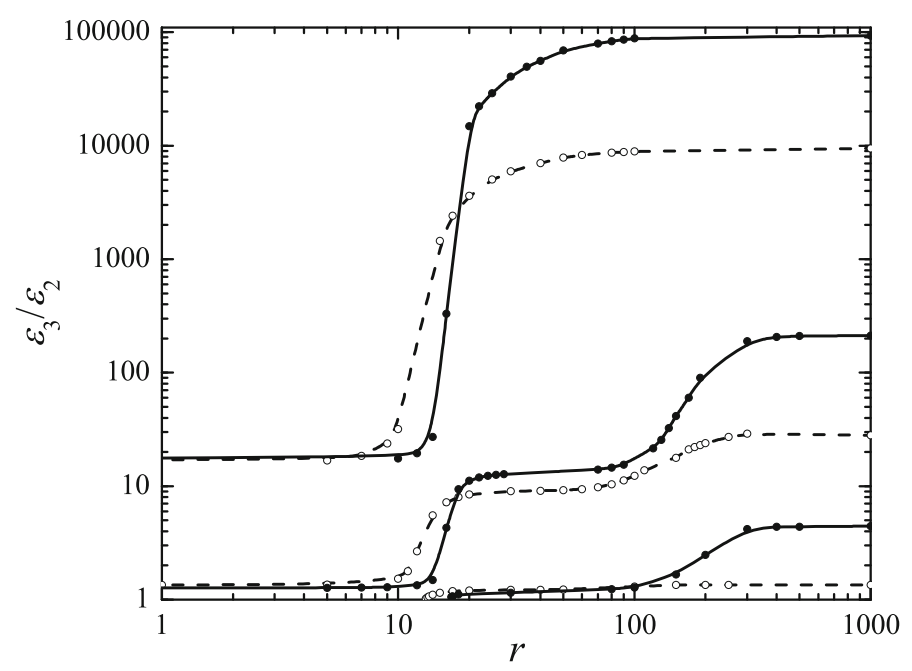

Fig. $2 \epsilon_{3} / \epsilon_{2}$ as a function of $r$, for $\epsilon_{2}=10^{-7}$ (fulled circles) and $10^{-6}$ (open circles). The solid and dashed lines are guides to the eye. As the $2 \mathrm{D}$ limit $(r \rightarrow 0)$ is approached, higher excited states disappear and only the ground and first excited states remain

Going to the 2D limit, it is important to notice that a quasi-2D system is in practice a 3D system. Then, the units of $\tau_{3 D}^{-1}(E)$ and $\tau_{p}^{-1}(E)$ are exactly the same, but are different from $\tau_{2 D}^{-1}(E)$. Taking into account the correct units, the 2D limit of Eq. (16) reads

$$
\tau_{2 D}^{-1}(E)=\lim _{R \rightarrow 0} R \tau_{p}^{-1}(E)=-2 \pi \ln \left(\sqrt{\frac{|E|}{\left|E_{2}\right|}}\right) .
$$

Expressions in Eqs. (17) and (18) are respectively identical to the expressions presented in Table 1 for 3D and 2D two-body T-matrix when $m_{a}=m_{b}=m_{c}$.

Introducing dimensionless variables, $\epsilon_{3}=E_{3} / \mu^{2}, \epsilon_{2}=E_{2} / \mu^{2}, r=R \mu, y_{\perp}=q_{\perp} / \sqrt{\mu}$ and $x_{\perp}=$ $k_{\perp} / \sqrt{\mu}$ and integrating over the angular dependence, since the focus is on states with zero angular momentum, the integral equation (15) is written as

$$
\begin{aligned}
f\left(y_{\perp}, n\right)= & \left\{\pi \ln \left[\frac{\sinh \left(\pi r \sqrt{\frac{3}{4}\left(y_{\perp}^{2}+\frac{n^{2}}{r^{2}}\right)-\epsilon_{3}}\right)}{\sinh \left(\pi r \sqrt{\epsilon_{2}}\right)}\right]\right]^{-1} \\
& \times \sum_{m=-\infty}^{\infty} \int_{0}^{\infty} d x_{\perp} x_{\perp} f\left(x_{\perp}, m\right)\left(\frac{1}{\sqrt{\left(-\epsilon_{3}+y_{\perp}^{2}+x_{\perp}^{2}+\frac{n^{2}}{r^{2}}+\frac{m^{2}}{r^{2}}+\frac{n m}{r^{2}}\right)^{2}-y_{\perp}^{2} x_{\perp}^{2}}}\right. \\
& \left.-\frac{1}{\sqrt{\left(1+y_{\perp}^{2}+x_{\perp}^{2}+\frac{n^{2}}{r^{2}}+\frac{m^{2}}{r^{2}}+\frac{n m}{r^{2}}\right)^{2}-x_{\perp}^{2} y_{\perp}^{2}}}\right) .
\end{aligned}
$$

The dimensional crossover transition is explored through the numerical solution of Eq. (19). In Fig. 2 the ratios $\epsilon_{3} / \epsilon_{2}$ are showed as function of the compact dimension radius $r$, for the ground, first, and second excited states. Notice that the last state goes into the continuum before the $2 \mathrm{D}$ limit is reached.

The computations were performed for two fixed two-body energies $\epsilon_{2}=10^{-6}$ (empty circles/dashed lines) and $10^{-7}$ (full circles/solid lines). Note that the Efimov ratio between two consecutive three-body states, $\sim 515$, is not completely reproduced for a finite $a$. The points at which the energies are calculated are showed explicitly, while the curves are guides to the eye and for $r=1000$ the energies are obtained from the pure 3D equation. 
An interesting dimensional crossover result is seen in Fig. 2, where only one sharp transition is present for the ground state while there are two for the first excited state. This behavior can be understood by considering the size of the trimer given roughly by $\bar{r} \sim 1 / \sqrt{\epsilon_{3}}$. For $\epsilon_{2}=10^{-7}$, the ground state plateau for $\epsilon_{3} / \epsilon_{2}=93330$ is placed at $\bar{r}=10.35$ and first excited state plateau for $\epsilon_{3} / \epsilon_{2}=211.79$ at $\bar{r}=217.29$. These $\bar{r}$ values give approximately the region of the jumps signaling that the $3 \mathrm{D}$ limit, represented by the plateau, is reached once the trimer size matches the size of the squeezed dimension, $r$. The same analysis can be made for $\epsilon_{2}=10^{-6}$ with $\bar{r}=10.27$ and $\bar{r}=188.98$, respectively, for the ground and first excited state. Varying $r$ from large to small values, the $3 \mathrm{D} \rightarrow 2 \mathrm{D}$ transition occurs for $r \sim 10$, where it is possible to notice the disappearance of the higher excited states in order to reproduce the well known 2D results with two trimer bound state energies proportional to $\epsilon_{2}$ with the ratios $\epsilon_{3} / \epsilon_{2}=16.52$ and $\epsilon_{3} / \epsilon_{2}=1.27$ [2].

From the experimental point of view it may be difficult to keep the dimer energy constant. However, the transition observed in Fig. 2 will not disappear due to a variation of $\epsilon_{2}$ with $r$. The increase of the dimer energy will merely move the beginning of the jumps towards smaller $r$. The optimal way to probe these jumps is to start from a two-body energy in the unitary limit $(a \rightarrow \infty)$ where the 2D plateaus are fixed. Larger dimer energies will cause the 3D plateau to move to lower $\epsilon_{3} / \epsilon_{2}$ ratio and push the beginning of the transition to smaller $r$, thus making the transition region broader. Another interesting study about the dimensional crossover, where three identical bosons are confined by a harmonic potential along one direction is found in [27].

\section{Conclusion}

In this paper we summarized our main results involving the single particle momentum distributions in two and three dimensions. The summary tables, comparing the matrix elements and the functional form for the different terms of the momentum distribution makes the comparison between the $2 \mathrm{D}$ and $3 \mathrm{D}$ regimes much easier. In the last section we presented in a very schematical form a method to continuously interpolate between different dimensions. The same technique used here to go from 3D to 2D, may be used to go from 2D to 1D systems. All discussions in this text followed closely the papers $[16,17,26]$. An interesting direction for future investigation, which connects all the results discussed in this work, is to understand whether the contact parameter tell us how much of the wave function is in each dimension in the transition region presented in Fig. 2 [13].

Acknowledgments We would like to thank Profs. Frederico, Zinner, Fedorov and Jensen for discussions. This work was partly supported by funds provided by the Brazilian agencies FAPESP (2013/04093-3), CNPq and CAPES (88881.030363/2013-01).

\section{References}

1. Landau, L.D., Lifshitz, E.M.: Quantum Mechanics. Pergamon Press, Oxford (1977)

2. Bruch, L.W., Tjon, J.A.: Binding of three identical bosons in two dimensions. Phys. Rev. A 19, 425-432 (1979)

3. Efimov, V.N.: Weakly-bound states of three resonantly-interacting particles. Yad. Fiz. 12, 1080 (1970)

4. Thomas, L.H.: The interaction between a neutron and a proton and the structure of $\mathrm{H}^{3}$. Phys. Rev. 47, $903-909$ (1935)

5. Kraemer, T., Mark, M., Waldburger, P., Danzl, J.G., Chin, C., Engeser, B., Lange, A.D., Pilch, K., Jaakkola, A., Nägerl, H.-C., Grimm, R.: Evidence for Efimov quantum states in an ultracold gas of caesium atoms. Nat. Phys. 440, 315-318 (2006)

6. Ferlaino, F., Grimm, R.: Forty years of Efimov physics: How a bizarre prediction turned into a hot topic. Phys. Online J. 3, 9 (2010)

7. Lim, T.K., Shimer, B.: The Fonseca-Redish-Shanley solvable model for a molecular three-body system and the efimov effect in two dimensions. Zeitschrift Fur Physik A Hadrons and Nuclei 297, 185-188 (1980)

8. Adhikari, S.K., Delfino, A., Frederico, T., Goldman, I.D., Tomio, L.: Efimov and Thomas effects and the model dependence of three-particle observables in two and three dimensions. Phys. Rev. A 37, 3666-3673 (1988)

9. Bellotti, F.F., Frederico, T., Yamashita, M.T., Fedorov, D.V., Jensen, A.S., Zinner, N.T.: Mass-imbalanced three-body systems in two dimensions. J. Phys. B Atomic Mol. Phys. 46(5), 055301 (2013)

10. Tan, S.: Energetics of a strongly correlated Fermi gas. Ann. Phys. 323(12), 2952-2970 (2008)

11. Kuhnle, E.D., Hu, H., Liu, X.-J., Dyke, P., Mark, M., Drummond, P.D., Hannaford, P., Vale, C.J.: Universal behavior of pair correlations in a strongly interacting fermi gas. Phys. Rev. Lett. 105(7), 070402 (2010)

12. Wild, R.J., Makotyn, P., Pino, J.M., Cornell, E.A., Jin, D.S.: Measurements of Tan's contact in an atomic Bose-Einstein condensate. Phys. Rev. Lett. 108(14), 145305 (2012)

13. Valiente, M., Zinner, N.T., Mølmer, K.: Universal properties of Fermi gases in arbitrary dimensions. Phys. Rev. A 86(4), 043616 (2012)

14. Bellotti, F.F., Frederico, T., Yamashita, M.T., Fedorov, D.V., Jensen, A.S., Zinner, N.T.: Dimensional effects on the momentum distribution of bosonic trimer states. Phys. Rev. A 87(1), 013610 (2013)

15. Castin, Y., Werner, F.: Single-particle momentum distribution of an Efimov trimer. Phys. Rev. A 83(6), 063614 (2011) 
16. Bellotti, F.F., Frederico, T., Yamashita, M.T., Fedorov, D.V., Jensen, A.S., Zinner, N.T.: Contact parameters in two dimensions for general three-body systems. N. J. Phys. 16(1), 013048 (2014)

17. Yamashita, M.T., Bellotti, F.F., Frederico, T., Fedorov, D.V., Jensen, A.S., Zinner, N.T.: Single-particle momentum distributions of efimov states in mixed-species systems. Phys. Rev. A 87, 062702 (2013)

18. Bellotti, F.F., Frederico, T., Yamashita, M.T., Fedorov, D.V., Jensen, A.S., Zinner, N.T.: Supercircle description of universal three-body states in two dimensions. Phys. Rev. A 85, 025601 (2012)

19. Danilov, G.S. : Zh. Eksp. Teor. Fiz 40, 698 (1961)

20. Bloch, I., Dalibard, J., Zwerger, W.: Many-body physics with ultracold gases. Rev. Mod. Phys. 80, 885-964 (2008)

21. Lamporesi, G., Catani, J., Barontini, G., Nishida, Y., Inguscio, M., Minardi, F.: Scattering in mixed dimensions with ultracold gases. Phys. Rev. Lett. 104, $153202(2010)$

22. Frederico, T., Delfino, A., Tomio, L., Yamashita, M.T.: Universal aspects of light halo nuclei. Prog. Part. Nucl. Phys. 67, 939994 (2012)

23. Stewart, J.T., Gaebler, J.P., Drake, T.E., Jin, D.S.: Verification of universal relations in a strongly interacting fermi gas. Phys. Rev. Lett. 104(23), 235301 (2010)

24. Fröhlich, B., Feld, M., Vogt, E., Koschorreck, M., Zwerger, W., Köhl, M.: Radio-frequency spectroscopy of a strongly interacting two-dimensional fermi gas. Phys. Rev. Lett. 106(10), 105301 (2011)

25. Werner, F., Castin, Y.: General relations for quantum gases in two and three dimensions. II. Bosons and mixtures. Phys. Rev. A 86(5), 053633 (2012)

26. Yamashita, M.T., Bellotti, F.F., Frederico, T., Fedorov, D.V., Jensen, A.S., Zinner, N.T.: Weakly bound states of two- and three-boson systems in the crossover from two to three dimensions. J. Phys. B Atomic Mol. Opt. Phys. 48(2), 025302 (2015)

27. Levinsen, J., Massignan, P., Parish, M.M.: Efimov trimers under strong confinement. Phys. Rev. X 4, 031020 (2014) 